\title{
Periodic flow of a second-grade fluid induced by non-torsional oscillations of eccentric rotating disks
}

\author{
H VOLKAN ERSOY \\ Department of Mechanical Engineering, Yildiz Technical University, 34349 Istanbul, Turkey \\ e-mail: hversoy@yildiz.edu.tr
}

MS received 17 December 2016; revised 27 May 2017; accepted 21 August 2017; published online 14 March 2018

\begin{abstract}
This paper deals with the periodic flow of a second-grade fluid caused by non-torsional oscillations of two disks rotating about non-coincident axes. While the two parallel disks are initially rotating with the same angular velocity about distinct axes, they start to execute non-torsional oscillations in their own planes and in the opposite directions. An exact solution is obtained for the components of the horizontal force per unit area exerted by the top and bottom disks on the fluid in the periodic state. The results are graphically displayed and the influence of the second-grade fluid parameter, the ratio of the frequency of oscillation to the angular velocity of the disks, the Reynolds number and the dimensionless velocity amplitudes of oscillation is discussed. It is observed that the change in the $x$-component of the mentioned force gets larger when the second-grade fluid parameter increases. However, an opposite effect is seen for the change in the $y$-component.
\end{abstract}

Keywords. Second-grade fluid; eccentric rotating disks; non-torsional oscillation; periodic flow.

\section{Introduction}

The flow of non-Newtonian fluids has gained considerable importance because of its applications in various branches of science, engineering and technology. Because of the inadequacy of the theory of Newtonian fluids in predicting the behaviour of some fluids, many constitutive equations that describe the non-Newtonian behaviour have been proposed. In the category of such fluids, the differential type fluids have acquired much popularity. There is a subclass of differential type fluids known as the second grade which has been extensively studied by the researchers. Although the second-grade fluids exhibit normal stress effects, they cannot predict the shear thinning and thickening effects. The second-grade fluids are dilute polymeric solutions (Khan et al [1] and Mohyuddin et al [2]).

The flow in the Maxwell orthogonal rheometer developed by Maxwell and Chartoff [3] has been studied during the past fifty decades. The rheometer consists of two parallel disks rotating with the same angular velocity about two non-coincident axes perpendicular to the disks. It was constructed to study polymer melts. Abbott and Walters [4] obtained an exact solution for the flow of a Newtonian fluid in this instrument. They also took into account the flow in the case of a viscoelastic fluid. Rajagopal [5] studied the flow of a second-order fluid between rotating coaxial and non-coaxial disks. Rajagopal and Gupta [6] established an exact solution for the flow of a second-grade fluid and studied the stability of this flow. Rajagopal [7] showed that the velocity field corresponding to the motion in an orthogonal rheometer is a motion with constant principal relative stretch history. In addition, we refer the reader to the papers by Rajagopal [8] and Srinivasa [9] for more details regarding the flow in an orthogonal rheometer.

Unsteady flows of a Newtonian fluid induced by oscillations of disks in an orthogonal rheometer have also received attention. Erdoğan $[10,11]$ studied the non-symmetrical unsteady flows due to the non-torsional oscillations of the disks while they are initially rotating about a common axis. In the first paper [10], he considered that the disks start to rotate eccentrically and the lower disk executes oscillations. In the second paper [11], he took into account that the disks start to rotate eccentrically and both the disks execute oscillations in the same direction. Ersoy [12] studied the unsteady symmetrical flow produced by the non-torsional oscillations of the disks in their own planes and in the opposite directions while they are initially rotating about non-coincident axes. Giri et al [13] investigated the flow induced by the effect of a magnetic field in the same geometry. Ersoy [14] examined the periodic flow induced by non-torsional oscillations of two porous disks in their own planes and in the opposite directions while they are initially rotating about two distinct axes. Recently, Ersoy [15] extended this study to the flow caused by the applied magnetic field. Further, the reader may consult the papers by Hayat et al $[16,17]$ for flows induced by noncoaxial rotation of a disk executing non-torsional oscillations and a second-grade fluid rotating at infinity. 
In this paper, the disks are initially rotating with the same speed about non-coincident axes. The fluid under consideration is a second-grade fluid; thus, the initial condition is the solution obtained by Rajagopal and Gupta [6]. The disks start to perform non-torsional oscillations in their own planes and in the opposite directions. After transients associated with the development from the initial state decay, the periodic motion of the fluid is studied. The symmetric condition is satisfied in view of the oscillations of the disks in the opposite directions. After an exact solution of the velocity field is obtained, a special attention is focused on the shear stresses since the study of the horizontal force applied by the fluid on the disks in an orthogonal rheometer is important. It is shown that the horizontal force on the upper disk is equal to that on the lower disk for the disks having the same radius. The influence of all the parameters on the flow is analysed and discussed through graphs in detail. The main aim of this paper is to study the influence of the non-Newtonian property of fluid. It is observed that the changes in the $x$ component of force get larger but those in the $y$-component get smaller with an increase in the second grade fluid parameter.

\section{Basic equations and solution}

The stress $\mathbf{T}$ in a homogeneous second-grade fluid is given by Rivlin and Ericksen [18] as

$$
\mathbf{T}=-p \mathbf{I}+\mu \mathbf{A}_{1}+\alpha_{1} \mathbf{A}_{2}+\alpha_{2} \mathbf{A}_{1}^{2},
$$

where $p$ is the pressure, I the identity tensor and $\mu$ the dynamic viscosity coefficient; $\alpha_{1}$ and $\alpha_{2}$ are the normal stress moduli. $\mathbf{A}_{1}$ and $\mathbf{A}_{2}$ stand for the first two RivlinEricksen tensors defined through

$$
\mathbf{A}_{1}=\operatorname{grad} \mathbf{v}+(\operatorname{grad} \mathbf{v})^{T}
$$

and

$$
\mathbf{A}_{2}=\dot{\mathbf{A}_{1}}+\mathbf{A}_{1}(\operatorname{grad} \mathbf{v})+(\operatorname{grad} \mathbf{v})^{T} \mathbf{A}_{1},
$$

where $\mathbf{v}$ is the velocity vector and the overdot denotes material time differentiation. According to Dunn and Fosdick [19], the second-grade fluid model is compatible with thermodynamics when the Helmholtz free energy of the fluid is a minimum for the fluid in equilibrium. The fluid then has general and pleasant boundedness and stability properties. The aforementioned situation and ClausiusDuhem inequality imply that the coefficients $\mu, \alpha_{1}$ and $\alpha_{2}$ must satisfy

$$
\mu \geq 0, \alpha_{1} \geq 0, \alpha_{1}+\alpha_{2}=0 .
$$

A comprehensive discussion on the restrictions for $\mu, \alpha_{1}$ and $\alpha_{2}$ can be found in the work by Dunn and Rajagopal
[20]. The sign of the material moduli $\alpha_{1}$ and $\alpha_{2}$ is the subject of much controversy. Fosdick and Rajagopal [21] showed that when $\alpha_{1}<0$, the fluid model considered leads to an unacceptable general instability.

Let us consider an incompressible second-grade fluid having the density $\rho$ between two disks located at $z=0$ and $z=h$. The top and bottom disks are initially rotating about the $z^{\prime}$ - and $z^{\prime \prime}$-axes with the same angular velocity $\Omega$, respectively. The eccentricity is along the $y$ axis and the distance between the axes of rotation is denoted by $a$. The top and bottom disks start to perform non-torsional oscillations in their own planes with the velocities $\mathbf{U}$ and $-\mathbf{U}$, respectively, where $\mathbf{U}=\left(U_{x} \mathbf{i}+U_{y} \mathbf{j}\right)$ sin $n t$. After the transients disappear, the periodic flow that occurs in the fluid is studied. The distance between the axes of rotation is fixed during the motion. The schematic configuration of the problem is illustrated in figure 1.

Therefore, the appropriate initial and boundary conditions for the velocity field are

$$
\begin{aligned}
& u=-\Omega(y-\hat{g}(z)), \quad v=\Omega(x-\hat{f}(z)) \\
& \text { at } t=0 \text { for } 0 \leq z \leq h,
\end{aligned}
$$

$$
\begin{aligned}
& u=-\Omega(y-a / 2)+U_{x} \sin n t, \quad v=\Omega x+U_{y} \sin n t \\
& \text { at } z=h \text { for } t \geq 0,
\end{aligned}
$$

$$
\begin{gathered}
u=-\Omega(y+a / 2)-U_{x} \sin n t, \quad v=\Omega x-U_{y} \sin n t \\
\text { at } z=0 \text { for } t \geq 0, \\
\quad u=-\Omega y, \quad v=\Omega x \text { at } z=h / 2 \text { for } t \geq 0 .
\end{gathered}
$$

The functions $\hat{f}(z)$ and $\hat{g}(z)$ represent the centres of rotation along $z$-direction corresponding to the steady flow between eccentric rotating disks for a second-grade fluid, and were obtained by Rajagopal and Gupta [6] as follows:

$$
\begin{aligned}
& \hat{f}(z)+i \hat{g}(z)=\frac{i a}{2} \\
& \quad \times\left[\frac{e^{(m+i n) z}-e^{-(m+i n) z}+e^{(m+i n)(z-h)}-e^{-(m+i n)(z-h)}}{e^{(m+i n) h}-e^{-(m+i n) h}}\right],
\end{aligned}
$$

where

$$
\begin{aligned}
m^{2}= & \frac{\rho\left(\sqrt{\mu^{2} / \Omega^{2}+\alpha_{1}^{2}}-\alpha_{1}\right)}{2\left(\mu^{2} / \Omega^{2}+\alpha_{1}^{2}\right)}, \\
n^{2}= & \frac{\rho\left(\sqrt{\mu^{2} / \Omega^{2}+\alpha_{1}^{2}}+\alpha_{1}\right)}{2\left(\mu^{2} / \Omega^{2}+\alpha_{1}^{2}\right)} .
\end{aligned}
$$

The initial and boundary conditions given by Eqs. (5)(8) suggest that the components of the velocity vector can be written as 


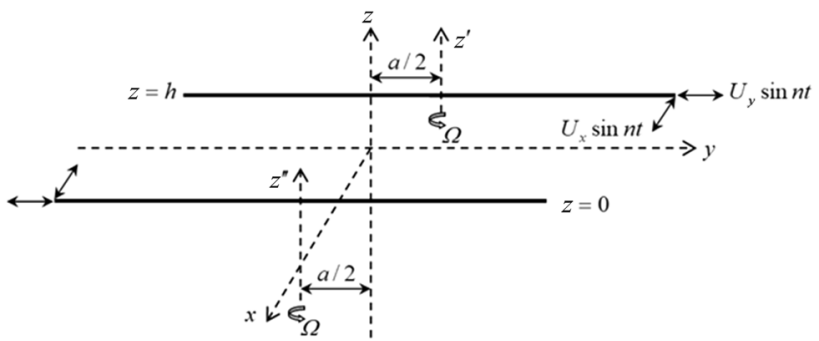

Figure 1. Flow geometry.

$$
u=-\Omega(y-g(z, t)), \quad v=\Omega(x-f(z, t)), \quad w=0,
$$

which satisfy the incompressibility condition.

Introducing $F(z, t)=f(z, t)+i g(z, t)$ and inserting Eqs. (1)-(3) and (11) into the equation of linear momentum (it is assumed that the body force is conservative), we obtain

$$
\begin{aligned}
\alpha_{1} & \Omega \frac{\partial^{3} F}{\partial t \partial z^{2}}+\left(\mu \Omega-i \alpha_{1} \Omega^{2}\right) \frac{\partial^{2} F}{\partial z^{2}}-\rho \Omega \frac{\partial F}{\partial t} \\
& -i \rho \Omega^{2} F=C(t),
\end{aligned}
$$

with

$$
\begin{gathered}
F(z, 0)=\hat{f}(z)+i \hat{g}(z), \\
F(h, t)=-\frac{U_{y}}{\Omega} \sin n t+i\left(\frac{a}{2}+\frac{U_{x}}{\Omega} \sin n t\right), \\
F(0, t)=\frac{U_{y}}{\Omega} \sin n t-i\left(\frac{a}{2}+\frac{U_{x}}{\Omega} \sin n t\right), \\
F\left(\frac{h}{2}, t\right)=0 .
\end{gathered}
$$

The symmetric condition shown by Eqs. (8) and (16) gives $C(t)=0$, which implies that the Poiseuille-type pressure gradient is zero during the motion. Introducing the dimensionless quantities

$$
\begin{aligned}
& \bar{F}(\zeta, \tau)=\frac{F(z, t)}{a}, \quad \zeta=\frac{z}{h}, \quad \tau=\Omega t, \quad \beta=\frac{\alpha_{1} \Omega}{\mu}, \\
& k=\frac{n}{\Omega}, \quad R=\frac{\rho \Omega h^{2}}{\mu}, \quad V_{x}=\frac{U_{x}}{\Omega a}, \quad V_{y}=\frac{U_{y}}{\Omega a}
\end{aligned}
$$

the governing Eq. (12) reduces to the following dimensionless form:

$$
\beta \frac{\partial^{3} \bar{F}}{\partial \tau \partial \zeta^{2}}+(1-i \beta) \frac{\partial^{2} \bar{F}}{\partial \zeta^{2}}-R \frac{\partial \bar{F}}{\partial \tau}-i R \bar{F}=0 .
$$

For the periodic motion in the fluid flow, it seems reasonable to suggest a solution of the form

$$
\bar{F}(\zeta, \tau)=\bar{F}_{0}(\zeta)+\bar{F}_{1}(\zeta) \cos k \tau+\bar{F}_{2}(\zeta) \sin k \tau,
$$

where $\bar{F}_{0}(\zeta)$ corresponds to the solution obtained by Rajagopal and Gupta [6]. Substituting Eq. (19) into Eq. (18), one obtains

$$
\begin{gathered}
(1-i \beta) \bar{F}_{0}^{\prime \prime}-i R \bar{F}_{0}=0 \\
(1-i \beta) \bar{F}_{1}^{\prime \prime}+k \beta \bar{F}_{2}^{\prime \prime}-i R \bar{F}_{1}-k R \bar{F}_{2}=0 \\
(1-i \beta) \bar{F}_{2}^{\prime \prime}-k \beta \bar{F}_{1}^{\prime \prime}-i R \bar{F}_{2}+k R \bar{F}_{1}=0,
\end{gathered}
$$

with

$$
\begin{gathered}
\bar{F}_{0}(1)=\frac{1}{2} i, \bar{F}_{0}(0)=-\frac{1}{2} i, \\
\bar{F}_{1}(1)=0, \bar{F}_{1}(0)=0, \\
\bar{F}_{2}(1)=-V_{y}+i V_{x}, \bar{F}_{2}(0)=V_{y}-i V_{x} .
\end{gathered}
$$

Thus, the solution corresponding to the translational velocity is

$$
\begin{aligned}
\bar{F}(\zeta, \tau)= & \frac{1}{2} i\left[\frac{1+\cosh P}{\sinh P} \sinh P \zeta-\cosh P \zeta\right] \\
& +\frac{1}{2}\left(V_{x}+i V_{y}\right)\left[\frac{1+\cosh D}{\sinh D} \sinh D \zeta-\cosh D \zeta\right. \\
& \left.+\cosh B \zeta-\frac{1+\cosh B}{\sinh B} \sinh B \zeta\right] \cos k \tau \\
& +\frac{1}{2}\left(-V_{y}+i V_{x}\right)\left[\frac{1+\cosh B}{\sinh B} \sinh B \zeta-\cosh B \zeta\right. \\
& \left.+\frac{1+\cosh D}{\sinh D} \sinh D \zeta-\cosh D \zeta\right] \sin k \tau
\end{aligned}
$$

where

$$
\begin{aligned}
P & =\sqrt{\frac{i R}{1-i \beta}}, \quad B=\sqrt{\frac{i R(1-k)}{1-i \beta(1+k)}} \\
D & =\sqrt{\frac{i R(1+k)}{1-i \beta(1-k)}}
\end{aligned}
$$

In each $z=$ constant plane, there exists a single point at which the velocity is zero. The locus of these points corresponding to the centres of rotation of the fluid layers is a curve described by $x=f(z, t)$ and $y=g(z, t)$ as the $z=$ constant plane shifts from $z=0$ to $z=h$. Figure 2 shows the locus of the centres of rotation as the parameters change. When the second-grade fluid parameter $(\beta)$ and the dimensionless velocity amplitudes of oscillation $\left(V_{x}, V_{y}\right)$ increase, the centres of rotation tend to move away from the $z$-axis. However, the mentioned points change their place periodically in the flow domain due to the non-torsional oscillations of the disks depending on the ratio of the frequency of oscillation to the angular velocity of the disks 

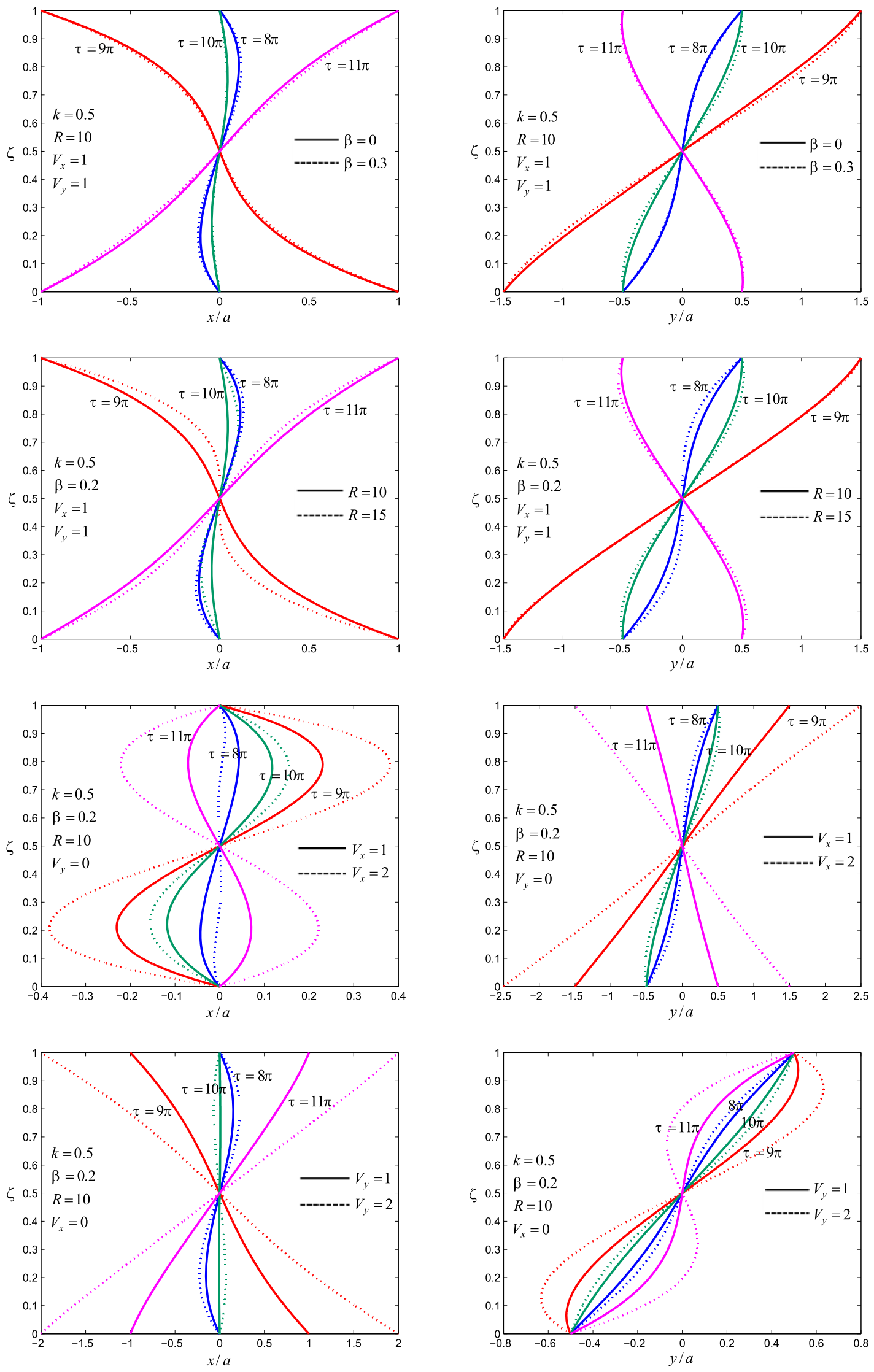

Figure 2. Locus of the points at which the velocity is zero. 
$(k)$. The points representing the centres of rotation in the core region become closer to the z-axis with the increase of the Reynolds number $(R)$. Thus, the flow has a boundarylayer structure.

The shear stress components $T_{x z}$ and $T_{y z}$ in the fluid are found in the complex form as follows:

$$
\begin{aligned}
\bar{T}_{x z} & +i \bar{T}_{y z}=\frac{1}{2}(1-i \beta) K_{1}(\zeta) \\
& +\frac{1}{2}\left(V_{x}+i V_{y}\right)\left[-(\beta+i) K_{2}(\zeta)+k \beta K_{3}(\zeta)\right] \cos k \tau \\
& +\frac{1}{2}\left(V_{x}+i V_{y}\right)\left[(1-i \beta) K_{3}(\zeta)+i k \beta K_{2}(\zeta)\right] \sin k \tau,
\end{aligned}
$$

where

$$
\begin{gathered}
\bar{T}_{x z}=\frac{T_{x z}}{\mu \Omega a / h}, \\
\bar{T}_{y z}=\frac{T_{y z}}{\mu \Omega a / h}, \\
K_{1}(\zeta)=P\left(\frac{1+\cosh P}{\sinh P} \cosh P \zeta-\sinh P \zeta\right), \\
K_{2}(\zeta)=D\left(\frac{1+\cosh D}{\sinh D} \cosh D \zeta-\sinh D \zeta\right) \\
+B\left(\sinh B \zeta-\frac{1+\cosh B}{\sinh B} \cosh B \zeta\right), \\
K_{3}(\zeta)=B\left(\frac{1+\cosh B}{\sinh B} \cosh B \zeta-\sinh B \zeta\right) \\
+D\left(\frac{1+\cosh D}{\sinh D} \cosh D \zeta-\sinh D \zeta\right) .
\end{gathered}
$$

Also, it should be noted that the following equalities should be used for the results in the limit $k \rightarrow 1$ $(n=\Omega)$ :

$$
\lim _{k \rightarrow 1} \frac{\sinh B \zeta}{\sinh B}=\zeta, \quad \lim _{k \rightarrow 1} \frac{\sinh B}{B}=1 .
$$

\section{Results and discussion}

The flow in the orthogonal rheometer executing non-torsional oscillations is a result of superposition of a rigid body rotation with the same angular velocity about the vertical axis passing the origin and a rigid body translation with non-torsional oscillation in each plane parallel to the disks. There is no flow perpendicular to the disks. It is clear that no torque is exerted by the fluid on the disk since the disks and fluid layers rotate with a common angular velocity. The measure of tangential force on one of the disks is particularly important. Thus, it is possible to determine the material moduli of a non-Newtonian fluid.

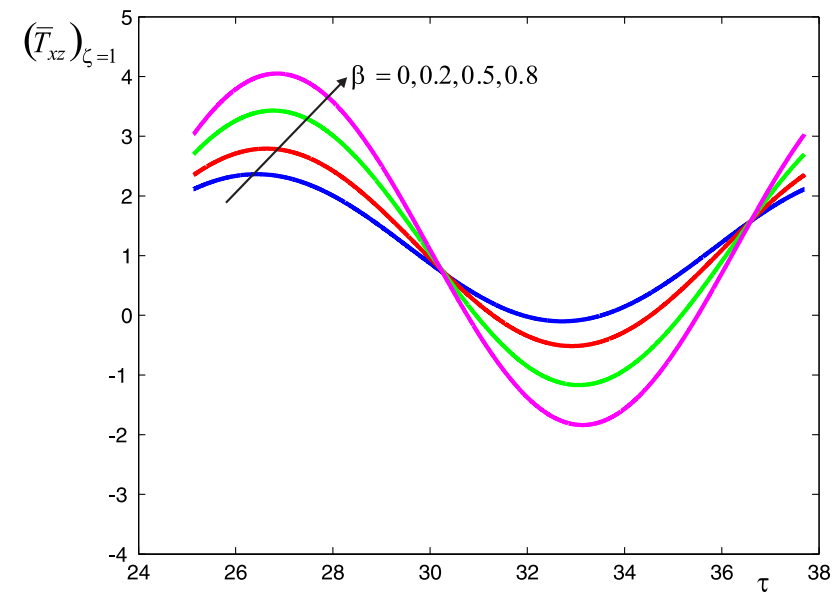

(a)

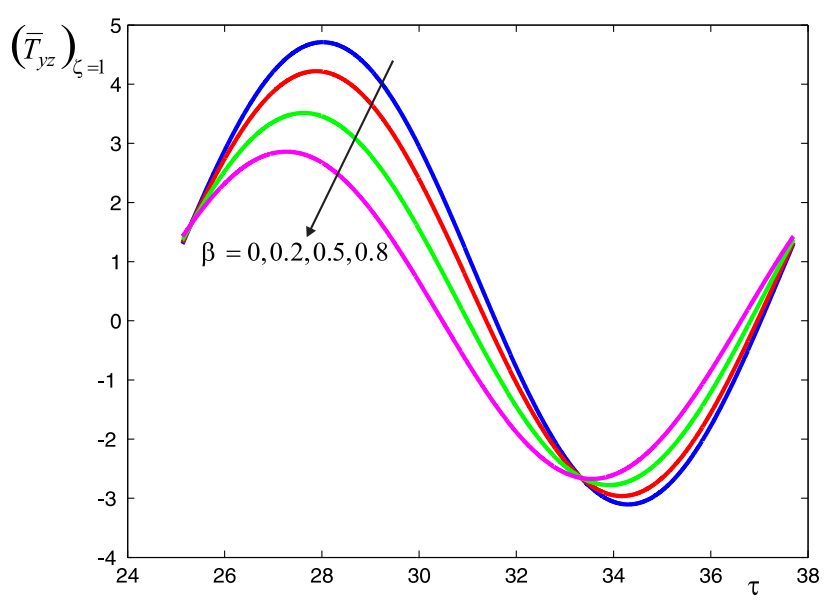

(b)

Figure 3. Effect of $\beta$ on $\left(\bar{T}_{x z}\right)_{\zeta=1}$ and $\left(\bar{T}_{y z}\right)_{\zeta=1}(\beta=0$ corresponds to Newtonian fluid) $\left(k=0.5, R=10, V_{x}=1, V_{y}=1\right)$.

This paper is concerned with the periodic flow of a second-grade fluid produced by non-torsional oscillations of the disks in an orthogonal rheometer. The disks are initially rotating with the same angular velocity about noncoincident axes normal to the disks. In other words, the initial condition is the solution obtained by Rajagopal and Gupta [6]. The disks suddenly start to perform non-torsional oscillations in their own planes and in the opposite directions. The sine oscillations of the disks are selected instead of the cosine oscillations that do not satisfy the initial condition. When the oscillation motion is set up, the flow contains transients for small times. After the initial transients die away, the motion of the fluid starts to be periodic in time. The beginning of the periodic motion in the fluid depends on the values of parameters. An examination of the paper by Ersoy [12] shows that the flow attains its periodic state at about the time $\tau=3,4,5$ for $k=0.5,1,1.25$, respectively, when $V_{x}=1, V_{y}=1$ and $R=10$. When the other parameters $\left(R, V_{x}, V_{y}\right)$ change in the appropriate interval, it is seen that the periodic flow 




(a)

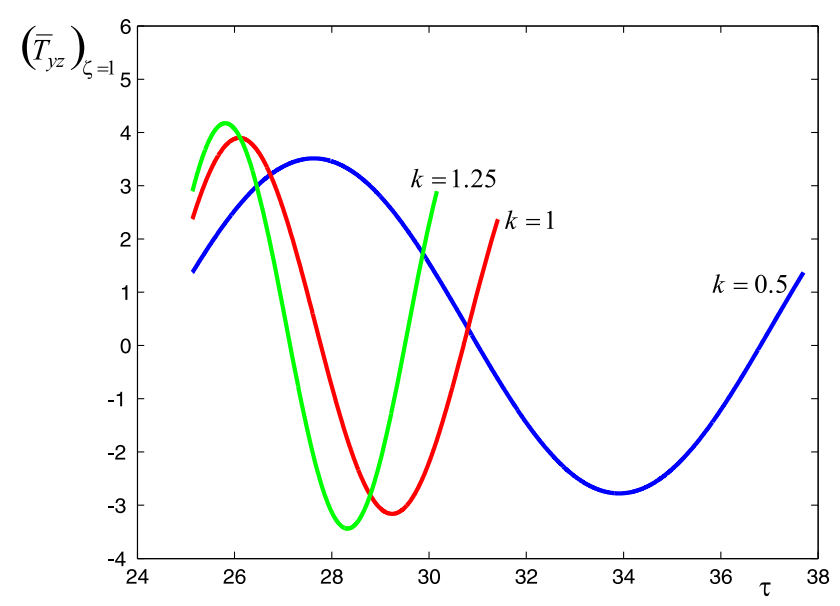

(b)

Figure 4. Effect of $k$ on $\left(\bar{T}_{x z}\right)_{\zeta=1}$ and $\left(\bar{T}_{y z}\right)_{\zeta=1}(\beta=0.5, R=10$, $\left.V_{x}=1, V_{y}=1\right)$.

starts much earlier than $\tau=8 \pi$ (In the mentioned paper, the disks are located at $z= \pm h$, the distance between the axes of rotation is $2 \ell$, and $V_{x}$ and $V_{y}$ are defined by $V_{x}=$ $U_{x} /(\Omega \ell)$ and $V_{y}=U_{y} /(\Omega \ell)$. The geometry in the present paper is the same as that used in the paper by Rajagopal and Gupta [6], who obtained the initial condition for the present paper). For convenience, the examination in this paper is made after $\tau=8 \pi$, at which the flow already attains its periodic state for the selected values of parameters $[14,15]$.

Since all the conditions are symmetric during the motion, the Poiseuille-type pressure gradient is zero. As a consequence of this, the shear stresses $T_{x z}$ and $T_{y z}$ on the top disk have the same values as those on the bottom disk, i.e., $\left(T_{x z}\right)_{z=h}=\left(T_{x z}\right)_{z=0}$ and $\left(T_{y z}\right)_{z=h}=\left(T_{y z}\right)_{z=0}$. The variations of the dimensionless shear stresses on the top disk $\left(\left(\bar{T}_{x z}\right)_{\zeta=1}\right.$ and $\left.\left(\bar{T}_{y z}\right)_{\zeta=1}\right)$ with the dimensionless time $(\tau)$ are investigated for all parameters with the help of figures 3, 4, 5, 6 and 7. Here, $\left(\bar{T}_{x z}\right)_{\zeta=1}$ and $\left(\bar{T}_{y z}\right)_{\zeta=1}$ represent the $x$ - and $y$ components of the dimensionless force per unit area exerted

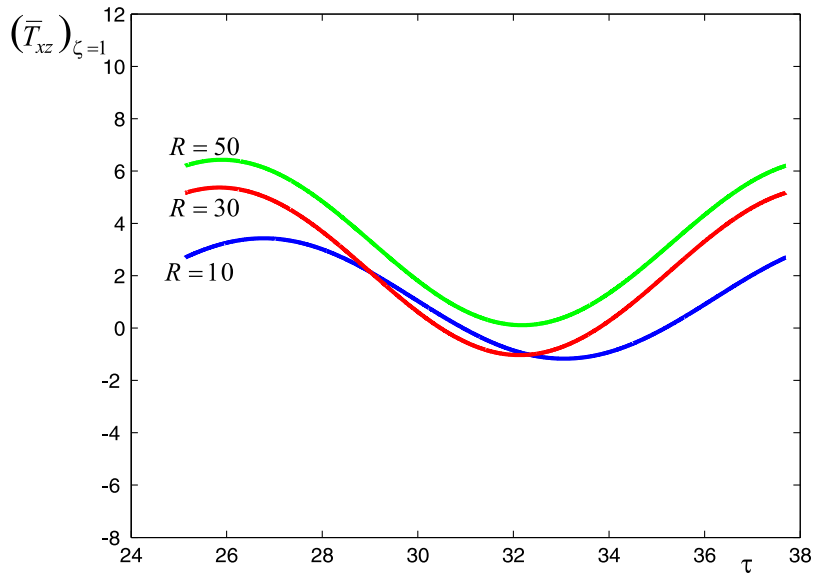

(a)



(b)

Figure 5. Effect of $R$ on $\left(\bar{T}_{x z}\right)_{\zeta=1}$ and $\left(\bar{T}_{y z}\right)_{\zeta=1}(\beta=0.5, k=0.5$, $\left.V_{x}=1, V_{y}=1\right)$.

by the top disk on the fluid, respectively. It is worth noting that the direction of the force exerted by the fluid is opposite to that exerted by the disk. Figure 3 shows the influence of the second-grade fluid parameter $(\beta)$. When the second-grade fluid parameter increases, the $x$-component varies with the larger amplitudes but an opposite effect is observed for the $y$-component. The effect of the ratio of the frequency of oscillation to the angular velocity of the disks $(k)$ is displayed in figure 4 . It is observed that the changes in the $x$ - and $y$-components become larger with its increase. Figure 5 indicates the effect of the Reynolds number $(R)$ based on the magnitude of the angular velocity. It is seen that an increase in the Reynolds number causes larger changes in the $x$ - and $y$-components. Figure 6 shows the variation of the dimensionless velocity amplitude along the direction perpendicular to the eccentricity $\left(V_{x}\right)$. The effect of the dimensionless velocity amplitude along the eccentricity direction $\left(V_{y}\right)$ is illustrated in figure 7 . It is seen that $V_{x}$ and $V_{y}$ lead to larger changes in the components of the force. 


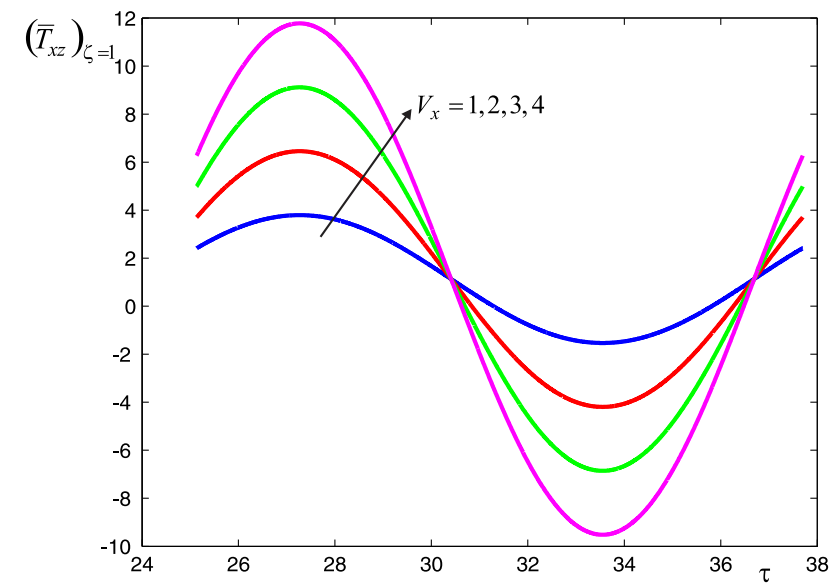

(a)

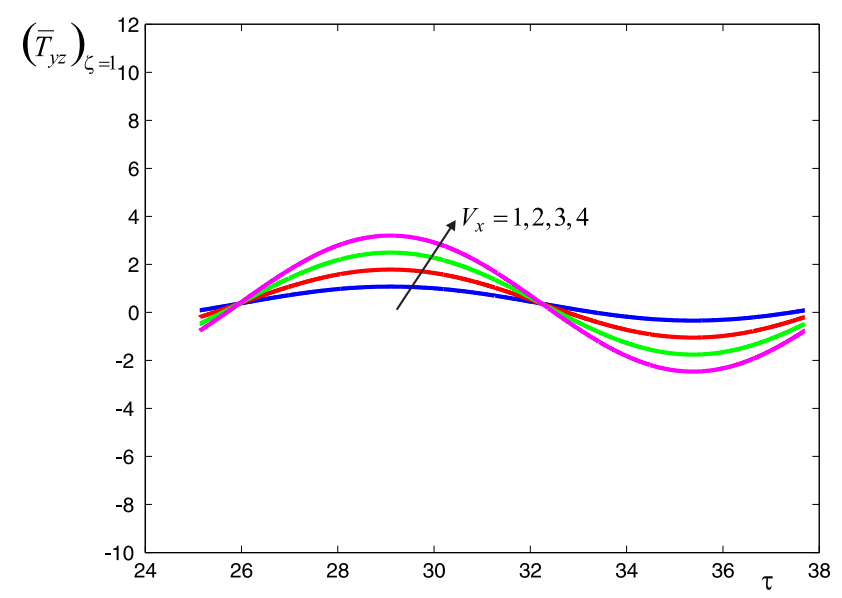

(b)

Figure 6. Effect of $V_{x}$ on $\left(\bar{T}_{x z}\right)_{\zeta=1}$ and $\left(\bar{T}_{y z}\right)_{\zeta=1} \quad(\beta=0.5$, $k=0.5, R=10, V_{y}=0$ ).

\section{Conclusions}

The main findings of the presented analysis can be summarized as follows:

- The increase of the second-grade fluid parameter gives rise to larger changes in the value of $\bar{T}_{x z}$ on the disks. On the other hand, its increase leads to a decrease of $\bar{T}_{y z}$ on the disks. The change in $\bar{T}_{y z}$ is more substantial than that in $\bar{T}_{x z}$ for a Newtonian fluid $(\beta=0)$. The flow is unaffected by the normal stress modulus $\alpha_{2}$, which is defined in the constitutive equation of a second-grade fluid.

- As ratio of the frequency of oscillation to the angular velocity of the disks increases, the amplitudes of $\bar{T}_{x z}$ and $\bar{T}_{y z}$ on the disks get larger.

- When the Reynolds number increases, the amplitudes of both $\bar{T}_{x z}$ and $\bar{T}_{y z}$ on the disks increase. The change in $\bar{T}_{y z}$ on the disks is larger than the change in $\bar{T}_{x z}$ on the disks. This is a consequence of the eccentricity.

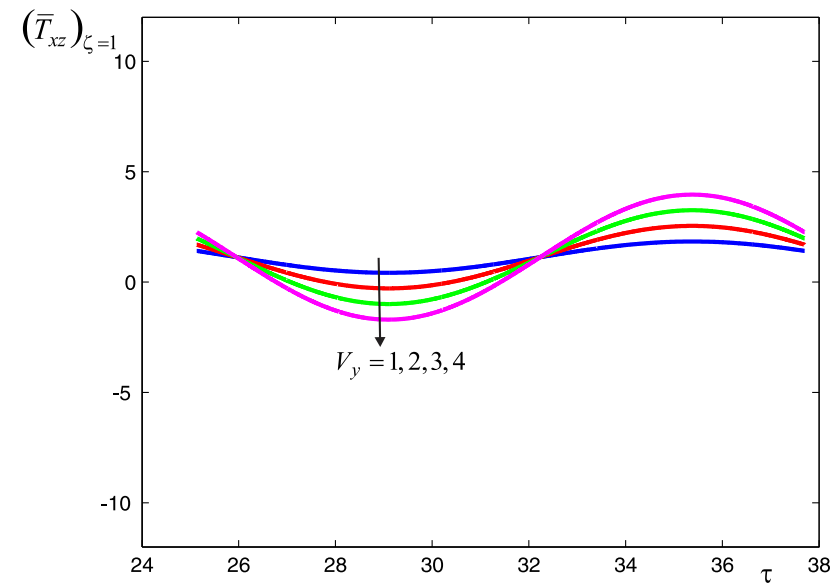

(a)

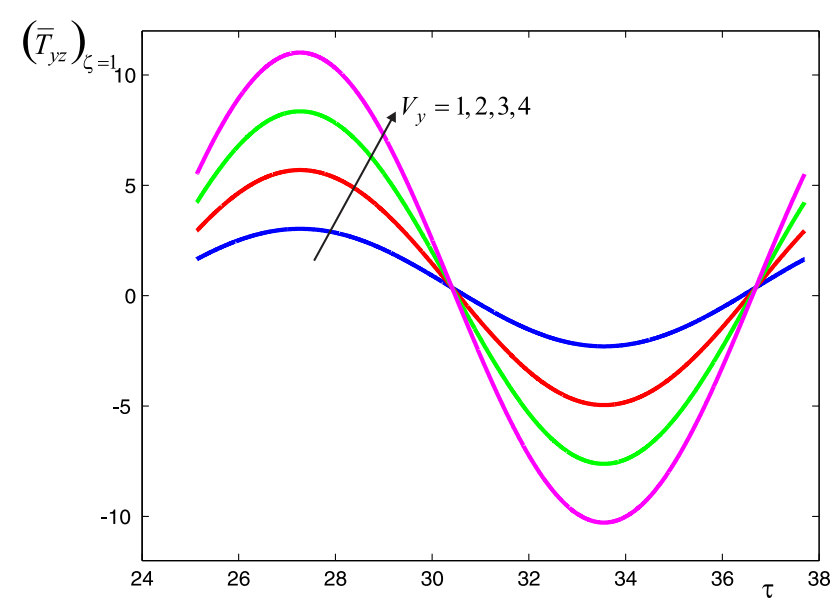

(b)

Figure 7. Effect of $V_{y}$ on $\left(\bar{T}_{x z}\right)_{\zeta=1}$ and $\left(\bar{T}_{y z}\right)_{\zeta=1}(\beta=0.5$, $k=0.5, R=10, V_{x}=0$ ).

- The increases in the dimensionless velocity amplitudes in the $x$ - and $y$-directions cause larger change in the values of $\bar{T}_{x z}$ and $\bar{T}_{y z}$ on the disks. When $V_{x}$ increases, it is seen that the change of $\bar{T}_{x z}$ on the disks is larger than that of $\bar{T}_{y z}$ on the disks. A reverse effect is noticed with an increase in $V_{y}$.

\section{Acknowledgements}

The author would like to express his sincere thanks to the referees for their valuable comments and suggestions.

\section{References}

[1] Khan M, Naheed E, Fetecau C and Hayat T 2008 Exact solutions of starting flows for second grade fluid in a porous medium. Int. J. Nonlin. Mech. 43(9): 868-879 
[2] Mohyuddin M R, Asghar S, Hayat T and Siddiqui A M 2006 On semi-inverse solutions for the time-dependent flows of a second-grade fluid. Math. Probl. Eng. 2006: 54295 (22 pp)

[3] Maxwell B and Chartoff R P 1965 Studies of a polymer melt in an orthogonal rheometer. Trans. Soc. Rheol. 9(1): $41-52$

[4] Abbott T N G and Walters K 1970 Rheometrical flow systems-part 2: theory for the orthogonal rheometer, including an exact solution of the Navier-Stokes equations. J. Fluid Mech. 40(1): 205-213

[5] Rajagopal K R 1981 The flow of a second order fluid between rotating parallel plates. J. Non-Newton. Fluid Mech. 9(1-2): 185-190

[6] Rajagopal K R and Gupta A S 1981 Flow and stability of a second grade fluid between two parallel plates rotating about noncoincident axes. Int. J. Eng. Sci. 19(11): 1401-1409

[7] Rajagopal K R 1982 On the flow of a simple fluid in an orthogonal rheometer. Arch. Rational Mech. Anal. 79(1): 39-47

[8] Rajagopal K R 1992 Flow of viscoelastic fluids between rotating disks. Theor. Comput. Fluid Dyn. 3(4): 185-206

[9] Srinivasa A R 2000 Flow characteristics of a multiconfigurational, shear thinning viscoelastic fluid with particular reference to the orthogonal rheometer. Theor. Comput. Fluid Dyn. 13(5): 305-325

[10] Erdoğan M E 1999 Flow due to parallel disks rotating about non-coincident axis with one of them oscillating in its plane. Int. J. Nonlin. Mech. 34(6): 1019-1030

[11] Erdoğan M E 2000 Unsteady flow between two eccentric rotating disks executing non-torsional oscillations. Int. J. Nonlin. Mech. 35(4): 691-699
[12] Ersoy H V 2012 Unsteady flow produced by oscillations of eccentric rotating disks. Math. Probl. Eng. 2012: 734784 (14 pp)

[13] Giri A, Das S and Jana R N 2014 Unsteady hydromagnetic flow due to oscillations of eccentric rotating disks. J. Nat. Sci. Sustain. Technol. 8(2): 237-258

[14] Ersoy H V 2015 Periodic flow due to oscillations of eccentric rotating porous disks. Adv. Mech. Eng. 2015: 1687814015599727 (8 pp)

[15] Ersoy H V 2017 Periodic flow due to non-torsional oscillations of eccentric rotating porous disks in the presence of a magnetic field. Mechanika 23(3): 397-401

[16] Hayat T, Ellahi R, Asghar S and Siddiqui A M 2004 Flow induced by non-coaxial rotation of a porous disk executing non-torsional oscillations and a second grade fluid rotating at infinity. Appl. Math. Model. 28(6): 591-605

[17] Hayat T, Ellahi R and Asghar S 2007 Unsteady magnetohydrodynamic non-Newtonian flow due to non-coaxial rotations of disk and a fluid at infinity. Chem. Eng. Commun. 194(1): 37-49

[18] Rivlin R S and Ericksen J L 1955 Stress-deformation relations for isotropic materials. J. Rational Mech. Anal. 4: 323-425

[19] Dunn J E and Fosdick R L 1974 Thermodynamics, stability and boundedness of fluids of complexity 2 and fluids of second grade. Arch. Rational Mech. Anal. 56(3): 191-252

[20] Dunn J E and Rajagopal K R 1995 Fluids of differential type: critical review and thermodynamic analysis. Int. J. Eng. Sci. 33(5): 689-729

[21] Fosdick R L and Rajagopal K R 1970 Anomalous features in the model of second order fluids. Arch. Rational Mech. Anal. 70(2): 145-152 